Título en Español:

UNA MIRADA AL MERCADEO EDUCATIVO

Título en Inglés:

A GLANCE AT EDUCATIONAL MARKETING

Autor:

Julián Eduardo Bucheli-Sandoval. $\operatorname{PhD}(c)^{1}$

Julio de 2020

Línea de profundización: Marketing Educativo

Grupo de Investigación Mercadeo I+2

Escuela de Marketing \& Branding

Institución Universitaria Politécnico Grancolombiano

\footnotetext{
${ }^{1}$ Estudiante del Doctorado en Ciencias de la Dirección de la Universidad del Rosario, Magister en Administración (MBA) y Magíster Mercadeo de la Universidad de Los Andes, Especialista en Marketing Estratégico de la Universidad del Valle y Administrador de Empresas de la Universidad del Cauca. Profesor Asociado de la Escuela de Marketing \& Branding del Politécnico Grancolombiano. Colombia. Correo-e: jebucheli@poligran.edu.co. ORCID: Orcid: https://orcid.org/0000-0001-8969-7606
}

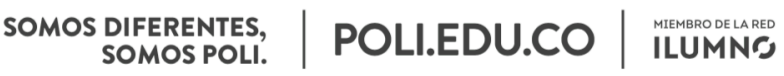




\title{
Resumen
}

El presente escrito pretender brindar una aproximación conceptual al estado del arte del mercadeo educativo, partiendo de la evolución histórica de la disciplina de marketing, recorriendo rápidamente las escuelas de pensamiento y nuevos paradigmas a fin de comprender la aplicación y relevancia de esta joven disciplina en el contexto de la educación terciaria o superior bajo la premisa de incitar a académicos e investigadores a involucrarse en este campo de conocimiento, el cual a pesar de haber tenido un crecimiento significativo durante estas tres últimas décadas, aun propone grandes retos en materia de teorización.

Palabras clave: Mercadeo educativo, evolución del mercadeo, escuelas de pensamiento de mercadeo.

\begin{abstract}
This paper aims to provide a conceptual approach to the state of the art of educational marketing, starting from the historical evolution of the marketing discipline, quickly going through the schools of thought and new paradigms in order to understand the application and relevance of this young discipline in the context of tertiary or higher education under the premise of inciting academics and researchers to get involved in this field of knowledge, which despite having had significant growth during these last three decades, still proposes great challenges in terms of theorizing.
\end{abstract}

Key words: Educational marketing, marketing evolution, marketing school of thinking. 


\section{Una Mirada al Mercadeo Educativo}

Desde de la década de 1970 se ha sostenido un sensible debate sobre la función principal ante la sociedad que deberían desempeñar las instituciones de educación terciaria o educación superior. Por una parte, hay quienes argumentan que debieran dedicarse exclusivamente a la creación de sabiduría y conocimiento (Newman \& Jahdi, 2009, Arum \& Roska, 2011), en tanto otros (Symes, 1998, Scott et al., 2004; Ball, 2004; Naidoo, 2007) indican que la creación de valor económico o utilidades es algo intrínseco que no puede obviarse y menos reprocharse considerando que las universidades modernas no solo expresan valores intelectuales y científicos, sino también valores sociales, culturales, instrumentales y organizacionales (Maringe \& Gibbs, 2009), a fin de adaptarse a las nuevas condiciones de un mercado y a garantizar su perdurabilidad empresarial.

La marketización o comercialización de la educación superior es el proceso de aplicación de las teorías económicas y de mercado a la planeación del sector académico (Hall, 2018), dada la creciente competencia de mercado en el sector de la educación terciaria, y los cambios en las composiciones demográficas, que impulsan cada vez más a las universidades a adoptar e implementar herramientas del management y el marketing para desarrollar ventajas competitivas que faciliten su diferenciación en el mercado, comprendiendo que las universidades pasaron de seccionar o escoger candidatos con alto potencial académico a luchar por convencer candidatos para reclutarlo y matricularlos en la oferta académica existente.

De acuerdo con Judson y Taylor (2014) y Hall (2018) las instituciones de educación superior adoptaron terminología, herramientas y modelos de negocio bajo la perspectiva de prestación de servicios educativos, en el cual se define el proceso de aprendizaje mediante la interacción entre oferentes y demandantes de conocimiento, en el cual, los profesores se 
cumplen el rol de proveedores y los estudiantes el de clientes, pero ambos deben operar bajo la perspectiva de co-producción de conocimiento (Gajda, 2009), a fin de garantizar el cumplimiento de una negociación responsable y ética implícita en el proceso de enseñanzaaprendizaje (Barnett, 2011).

A fin de comprender más a profundidad el concepto y estado actual de la marketización de la educación superior, es relevante conocer y comprender el transitar evolutivo del marketing como disciplina y las correspondientes escuelas de pensamiento que han dado origen a diversas vertientes y corrientes actuales de investigación y aplicación como el mercadeo educativo.

De acuerdo con Maclaran et al. (2011) los desarrollos teóricos son esenciales para cualquier disciplina, no solo para la creación de nuevo conocimiento, sino también para el establecimiento de su estado del arte, así mismo, permite identificar con cierta facilidad los vacíos en el conocimiento y las tendencias en investigaciones futuras, no obstante, para comprender los desarrollos en la el proceso de teorización del marketing es necesario comprender los debates filosóficos, económicos y sociológicos que han tenido lugar e influencia el desarrollo de esta joven disciplina.

Diversos debates han marcado el desarrollo del pensamiento en mercadeo, uno de ellos se centra en su esencia misma, es decir, si el mercadeo debería considerarse como ciencia o como arte, Hutchinson (1952) afirmaba que el mercadeo debía ser concebido como un arte, dado que dependía de la intuición y habilidades del dirigente, en tanto Hunt (1976) defendió la postura de ver el mercadeo bajo una óptica de carácter científico, dada la vinculación de herramientas cuantitativas y tecnológicas que permitieron procesamientos de información más complejos. 
El otro debate desarrollado se centró en el posicionamiento filosófico y epistemológico de la disciplina, dentro del cual se pueden mencionar posturas como: crítica relativista (Anderson, 1983), humanista (Monieson, 1988), pluralista (Siegel, 1988), postmodernista (Sherry, 1991; Brown, 1995), crítica realista (Easton, 2002), y postcolonialista (Jack, 2008) entre otras tantas. Estos debates epistemológicos han generado diversas clasificaciones de corrientes o escuelas de pensamiento a pesar de la discontinuidad o trazabilidad histórica, la abundancia de técnicas y la carencia de teorías que las respalden, de las diferencias teóricas, conceptuales y terminológicas de la disciplina y por último, la pluralización de las fuentes de difusión de información (Brownlie et al., 1999).

En la Figura 1 diseñada a partir de Shaw et al. (2011), presenta las primeras escuelas de marketing, las cuales se centraron en responder preguntas como, qué, cómo, quién y dónde, necesarias para describir y explicar la disciplina como un campo de estudio científico.

\section{Figura 1}

Primeras Escuelas de Pensamiento en Marketing

\begin{tabular}{|c|c|c|c|}
\hline \multicolumn{4}{|c|}{ Escuelas Iniciales } \\
\hline 1910 & & & 1970 \\
\hline $\begin{array}{l}\text { Escuela de Funciones } \\
\text { de Marketing }\end{array}$ & $\begin{array}{c}\text { Escuela de Productos } \\
\text { Genéricos }\end{array}$ & $\begin{array}{c}\text { Escuela de } \\
\text { Instituciones }\end{array}$ & $\begin{array}{l}\text { Escuela de comercio } \\
\text { interregional }\end{array}$ \\
\hline $\begin{array}{l}\text { ¿Cuál es el trabajo de } \\
\text { marketing? } \\
\text { Identificar y catalogar los } \\
\text { elementos fundamentales } \\
\text { del campo. } \\
\text { Las funciones son } \\
\text { "universales" } \\
\text { Converse (1945) }\end{array}$ & $\begin{array}{c}\text { ¿Cómo se comercializan } \\
\text { las diferentes clases de } \\
\text { bienes? } \\
\text { La escuela de productos } \\
\text { básicos se centró en las } \\
\text { características distintivas } \\
\text { de los bienes (es decir, } \\
\text { productos y servicios) } \\
\text { Copeland (1924) }\end{array}$ & $\begin{array}{l}\text { ¿Hay demasiados } \\
\text { intermediarios? " } \\
\text { Las instituciones de } \\
\text { marketing se refieren a } \\
\text { aquellos que hacen el } \\
\text { trabajo de marketing, } \\
\text { generalmente } \\
\text { intermediarios de } \\
\text { marketing, incluidos } \\
\text { mayoristas, agentes, } \\
\text { corredores y minoristas. } \\
\text { Beckman (1937); Bartels } \\
\text { (1988) }\end{array}$ & $\begin{array}{c}\text { ¿Dónde se lleva a cabo el } \\
\text { marketing? } \\
\text { Soportada en cuatro } \\
\text { premisas fundamentales: } \\
\text { (1) escasez de recursos, } \\
\text { (2) afluencia regional, (3) } \\
\text { demanda recíproca entre } \\
\text { regiones y (4) } \\
\text { competencia relativa } \\
\text { dentro de las regiones. } \\
\text { Reilly's (1931); Grether } \\
\text { (1950) }\end{array}$ \\
\hline
\end{tabular}

Entre tanto, en la Figura 2 diseñada a partir de Jones et al. (2011), presenta las escuelas modernas de marketing, las cuales centraron su atención en las funciones, las 
instituciones, el mercado y sus actores y las implicaciones que el marketing tiene con la sociedad y la sociedad con el marketing.

Figura 2

Escuelas Modernas de Pensamiento en Marketing

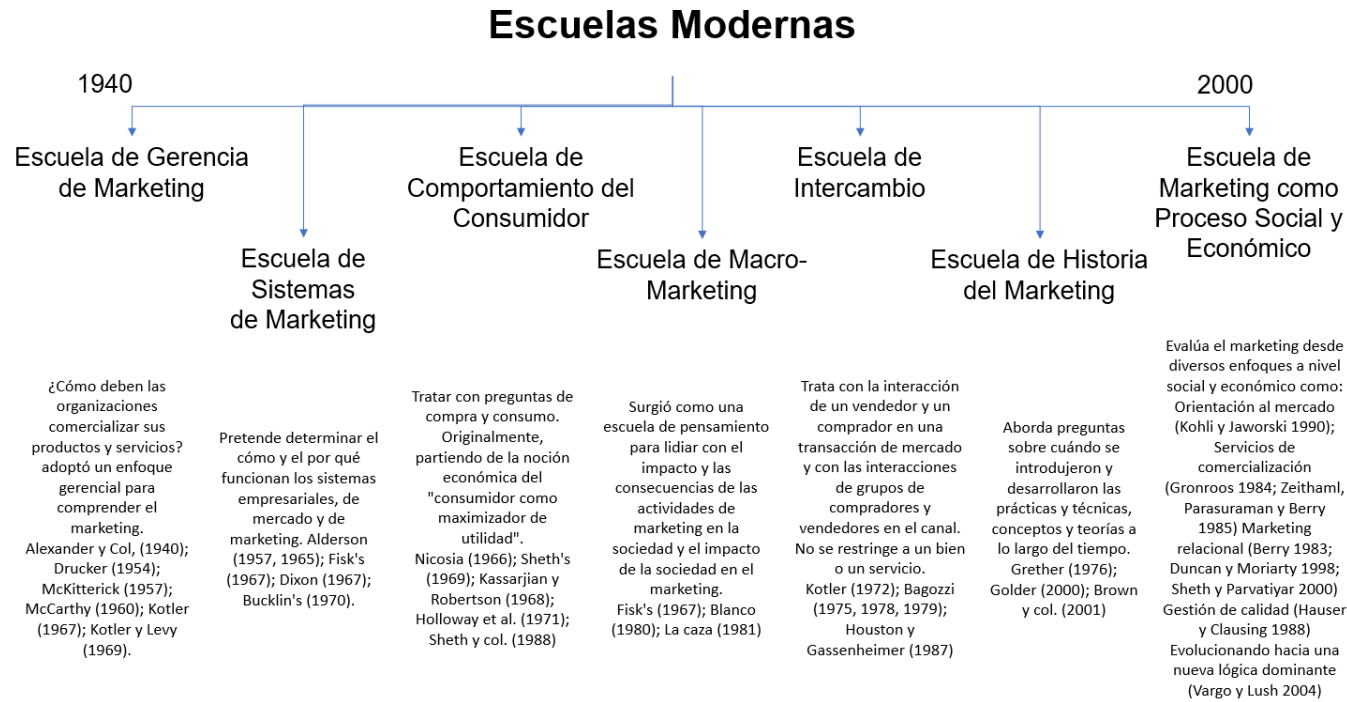

Bajo la comprensión de estas diferentes corrientes de pensamiento, es posible afirmar que la vertiente del mercadeo educativo parte de la Escuela de Gerencia de Marketing, dada la imperiosa necesidad de concebir las instituciones de educación como organizaciones y de cómo estas deberían comercializar sus productos educativos, para lo cual requieren de los principios de la gerencia, de sus decisiones, modelos, estrategias, técnicas, acciones y por supuesto de líderes que efectúen todo el proceso de planificación requerido para ejecutarlas.

Así las cosas, es en este nivel académico, el terciario, en el que surgen por primera vez en la década de 1970, las ideas del mercadeo educativo bajo dos corrientes, la norteamericana, con el desarrollo e intercambio de prácticas de reclutamiento de estudiantes entre las diversas instituciones de educación superior y la inglesa, con el reconocimiento de la importancia que tienen las relaciones públicas para la gestión de recursos (Foskett, 2012).

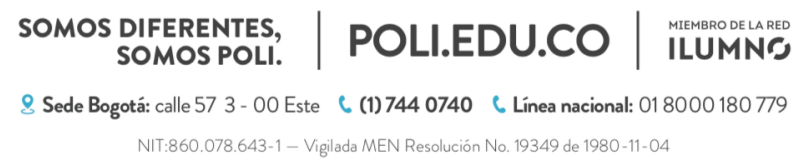


En la literatura regulada, el concepto de mercadeo educativo se presenta como las prácticas, métodos y herramientas aplicadas a las instituciones de educación superior para garantizar su supervivencia, prosperidad, competitividad y perdurabilidad en entornos altamente competitivos, especializados y globalizados (Stachowski, 2011). Como pioneros en la literatura se pueden mencionar a diversos autores, entre ellos, Krachenberg (1972) quien proporciona un marco de acción para comprender las necesidades de los alumnos y luego desarrollar ofertas para satisfacer dichas necesidades, Keen y Greenall (1987) quienes abordan el tema de la administración de relaciones públicas en universidades, Jonathan (1990) quien propone una adaptación de conceptos de mercado al sector educativo, Hunter (1991), Gewirtz et al. (1995) y Reay (1998), quien se focalizaron en investigar e identificar y priorizar factores de elección de los estudiantes y finalmente, Kotler y Fox (1995) a quienes podría considerarse como los autores más recordados gracias a su libro seminal denominado Strategic Marketing for Educational Institutions publicado en 1995, el cual aborda el mercadeo educativo como una tendencia emergente y se autodenomina como el manual para académicos y profesionales que desean mejorar la efectividad de las prácticas de mercadeo en las instituciones de educación superior.

La evolución de la literatura de mercadeo educativo ha tenido un evidente y sobresaliente desarrollo desde el contexto de educación superior, así mismo, ha primado copiosamente a nivel conceptual y empírico la perspectiva de la demanda, identificando mayoritariamente las expectativas de prospectos y estudiantes sobre sus necesidades, deseos, percepciones y satisfacción (Stachowski, 2011) como se evidencia en la Figura 3. 
Figura 3

Evolución del Mercadeo Educativo

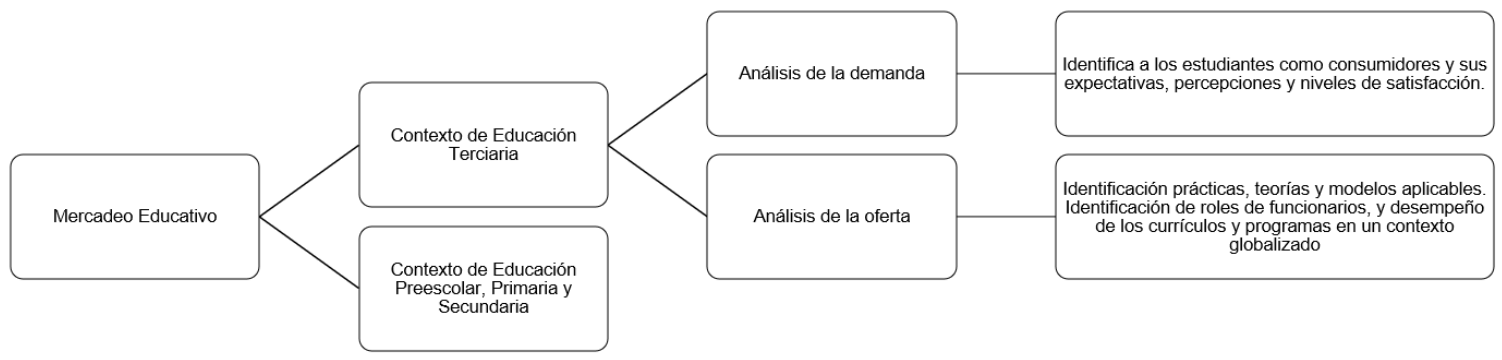

Los principales vacíos de conocimiento en el mercadeo educativo se encuentran a nivel de la perspectiva de la oferta, dado que las aplicaciones de técnicas, modelos y prácticas de marketing no son sistemáticos ni estandarizados y dependen de factores sociales, económicos, políticos, ambientales y culturales, en otras palabras, las prácticas que funcionaron en determinadas instituciones y naciones, no necesariamente funcionarán en otras latitudes, por tanto, las investigaciones para validación de prácticas, modelos e implicaciones demás dejan un campo amplio de acción a los investigadores (Hall y Witek, 2015).

Para finalizar, se pueden mencionar algunos tópicos que la Asociación Americana de Marketing ha propuesto como las principales tendencias en términos de mercadeo de instituciones de educación superior y que coinciden con la perspectiva de la oferta como son prácticas de reclutamiento y admisión orientadas desde las páginas web, redes sociales y motores de búsqueda, decisiones soportadas desde la analítica de datos, construcción de experiencias de alto impacto mediante acciones focalizadas, únicas y personalizadas y acciones de branding integrado a la identidad institucional, la estrategia corporativa y la experiencia académica de los estudiantes (AMA,2020). 


\section{Referencias}

AMA. (2020). 2020 Higher Ed Marketing Trends Roundup. American Marketing Association. https://www.ama.org/2020/01/14/2020-higher-ed-marketing-trends-roundup/

Anderson, P.F. (1983) 'Marketing, Scientific Progress, and the Scientific Method', Journal of Marketing, 47(4): 18-31. http://dx.doi.org/10.2307/1251395

Arum, R., \& Roksa, J. (2011). Academically adrift: Limited learning on college campuses. Chicago: The University of Chicago Press.

Ball, S.J. (2004) Education for Sale! Commodification of everything? Annual Education Lecture at the Karl Mannheim Professor of Sociology of Education Institute of Education, University of London.

Barnett, R. (2011). The marketised university: defending the indefensible. In M. Molesworth, R. Scullion, \& E. Nixon (Eds.), The marketisation of higher education and the student as consumer. London \& New York: Routledge Taylor \& Francis Group.

Brown, S. (1995) Postmodern Marketing. London: Routledge.

Brownlie, D., Saren, M., Wensley, R. and Whittington, R. (eds) (1999) Rethinking Marketing: Towards Critical Marketing Accountings. London: Sage.

Easton, G. (2002) 'Marketing: A Critical Realist Approach', Journal of Business Research, 55(2): 103-109. http://dx.doi.org/10.1016/S0148-2963\%2800\%2900145-4

Foskett, N. (2012). Marketisation and education marketing: The evolution of a discipline and a research field. En The Management and Leadership of Educational Marketing: Research, Practice and Applications (Vol. 15, pp. 39-61). Emerald Group Publishing Limited. https://doi.org/10.1108/S1479-3660(2012)0000015004

Gajda, A. (2009). The trials of academe the new era of campus litigation. New York: Harvard University Press. 
Gewirtz, S., Ball, S., \& Bowe, R. (1995). Markets, choice and equity in education. Milton Keynes, UK: Open University Press

Hall, H. (2018). The marketisation of higher education: symptoms, controversies, trends. Ekonomia i Prawo. Ecomomics and Law, 17(1): 33-42. http://dx.doi.org/10.12775/EiP.2018.003

Hall, H., \& Witek, L. (2016). Conditions, contemporary importance and prospects of higher education marketing on the example of Polish universities. Procedia Economics and Finance, 39, 206-211.

Hunt, S.D. (1976) 'The Nature and Scope of Marketing', Journal of Marketing, 40(3): 17-26. http://dx.doi.org/10.2307/1249990

Hunter, J. B. (1991). Which school? A study of parents' choice of secondary school. Educational Research, 33(1), 31-41.

Hutchinson, K.D. (1952) 'Marketing as Science: An Appraisal', Journal of Marketing, 16(January): 286-292. http://dx.doi.org/10.2307/1247541

Jack, G. (2008) 'Postcolonialism and Marketing', in M.Tadajewski and D.Brownlie (eds) Critical Marketing: Issues in Contemporary Marketing, pp. 363-384. Chichester: John Wiley.

Jonathan, R. (1990). State education or prisoner's dilemma. The 'hidden hand' as a source of education policy. Educational Philosophy and Theory, 22(1), 16-24.

Jones, B., Shaw, E., \& McLean, P. (2011). The Modern Schools of Marketing Thought. En The SAGE Handbook of Marketing Theory (SAGE Publications Ltd, pp. 45-28).

Judson, K.M., \& Taylor, S.A. (2014). Moving from marketization to marketing of higher education: the co-creation of value in higher education. Higher Education Studies, 4(1). DOI:10.5539/hes.v4n1p51 
Keen, C., \& Greenall, J. (1987). Public relations management in colleges, Polytechnics and universities. Banbury, UK: Heist Publications

Kotler, P., \& Fox, K. (1995). Strategic marketing for educational institutions (2nd ed.). Englewood Cliffs, CA: Prentice Hall.

Krachenberg AR (1972) Bringing the concept of marketing to higher education. Journal of Higher Education 43: 369-380.

Maclaran, P., Saren, M., Stern, B., \& Tadajewski, M. (2011). Introduction. En The SAGE Handbook of Marketing Theory (SAGE Publications Ltd, pp. 1-24).

Maringe, F., \& Gibbs, P. (2009). Marketing higher education. Maidenhead, UK: McGraw Hill.

Monieson, D.D. (1988) 'Intellectualization in Marketing: A World Disenchanted', Journal of Macromarketing, 8(2): 4-10. http://dx.doi.org/10.1177/027614678800800202

Naidoo, R. (2007) HE as a global commodity, Observatory on Borderless Higher Education.

Newman, S., \& Jahdi, K. (2009). Marketization of education: Marketing, rhetoric, and reality. Journal of Further and Higher Education, 33(1). https://doi.org/10.1080/03098770802638226

Reay, D. (1998). Setting the agenda; the growing impact of market forces in pupil grouping in British secondary schools. Journal of Curriculum Studies, 30(5), 545-558.

Scott, D., Brown, A., Lunt, I. and Thorne, L. (2004) Professional Doctorates: Integrating Professional and Academic Knowledge. Milton Keynes: Open University Press.

Shaw, E., Jones, B., \& McLean, P. (2011). The Early Schools of Marketing Thought. En The SAGE Handbook of Marketing Theory (SAGE Publications Ltd, pp. 27-41).

Sherry, J.F. (1991) 'Postmodern Alternatives The Interpretive Turn in Consumer Research', in T.S.Robertson and H.H.Kassarjian (eds) Handbook of Consumer Research, pp. 548-591. Englewood Cliffs: Prentice-Hall. 
Sheth, J.N. (1992) 'Acrimony in the Ivory Tower: A Retrospective on Consumer Research', Journal of the Academy of Marketing Science, 20(4): 345-353. http://dx.doi.org/10.1007/BF02725211

Siegel, H. (1988) 'Relativism for Consumer Research (Comments on Anderson)', Journal of Consumer Research, 15(1): 129-132. http://dx.doi.org/10.1086/209152

Stachowski, C. A. (2011). Educational Marketing: A Review and Implications for Supporting Practice in Tertiary Education. Educational Management Administration \& Leadership. https://doi.org/10.1177/1741143210390056

Symes, C. (1998). Education for sale: A semiotic analysis of school prospectuses and other forms of educational marketing. Australian Journal of Education, 42(2), 133-152. 